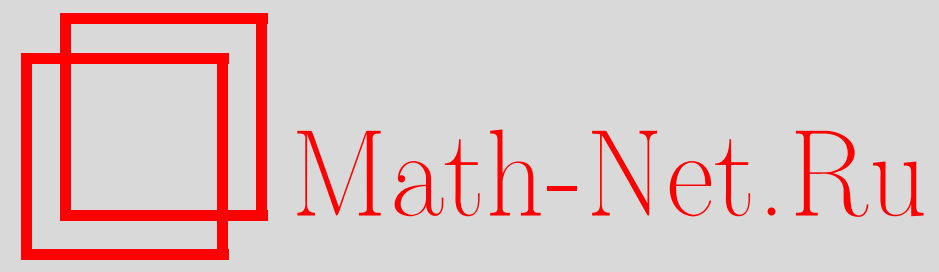

Э. М. Кудлаев, Слабая сходимость распределений разделимых статистик, Теория вероятн. и ее примен., 1997, том 42, выпуск 1, 85-107

DOI: https://doi.org/10.4213/tvp1714

Использование Общероссийского математического портала Math-Net.Ru подразумевает, что вы прочитали и согласны с пользовательским соглашением http://www. mathnet.ru/rus/agreement

Параметры загрузки:

IP : 54.198 .67 .100

26 апреля 2023 г., 12:22:09

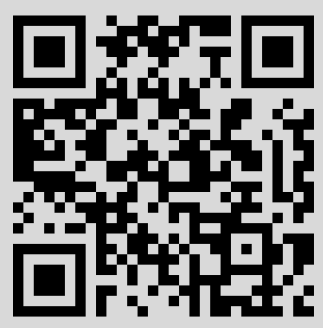




\section{СЛАБАЯ СХОДИМОСТЬ РАСПРЕДЕЛЕНИЙ РАЗДЕЛИМЫХ СТАТИСТИК ${ }^{1)}$}

Изучаются слабые пределы (с ростом числа слагаемых) распределений разделимых статистик со значениями в локально компактных хаусдорфовых абелевых группах. Рассматривается универсальный метод получения предельных теорем для разделимых статистик, приспособленный для всех схем. Полученный слабый предел выражается через предельное условное распределение сопутствуюшей разделимой статистике аддитивной формы, построенной по независимым случайным величинам.

Ключевые слова и фразы: разделимые статистики со значениями в локально компактных хаусдорфовых абелевых группах; теорема двойственности Л. С. Понтрягина; непрерывная версия регулярного условного распределения; интегральное представление Зейбелла для условной характеристической функции; суммирование независимых случайных величин; общий принцип сходимости Б. М. Клосса.

В настоящей работе изучаются слабые пределы (с ростом числа слагаемых) распределений сумм зависимых специальным образом случайных величин со значениями в локально компактных хаусдорфовых абелевых группах (LCA-группах, для краткости). Предполагается, что слагаемые таких сумм являются функпиями от случайных аргументов; последние являются зависимыми таким образом, что их совместное распределение совпадает с условным распределением некоторых независимых случайных величин при условии фиксации суммы последних в некоторой точке «a». В отечественной литературе суммы такого вида называются разделимыми статистиками (РС, для краткости) и широко используются как в математической статистике и теории вероятностей, так и в других областях математики (см. [3], [6], [10], [14], [15], [17], $[18],[38]$ ). Имеется ряд методов (см. [6], [9]-[12], [14], [18], [25], [26], [31], [34]-[36], [39], [41]) получения предельных теорем для РС. Здесь рассматривается универсальный способ, приспособленный для $\mathrm{PC}$ всех схем;

\footnotetext{
*Московский государственный университет им. М. В. Ломоносова, механихоматематический факультет, лаборатория математичесхой статистики, Воробьевы горы, 119899 Москва, Россия.

1) Работа выполнена при финансовой подцержке Российского фонда фундаментальных исследований, грант № 94-01-01273 и грантового центра по исследованиям в области математики при Новосибирском государственном университете.
} 
полученный при этом слабый предел распределения выражается через предельное условное распределение сопутствуюшей $\mathrm{PC}$ аддитивной формы, построенной по независимым случайным величинам.

Работа состоит из трех параграфов. Первый из них носит вспомогательный характер: в нем приводятся определения и обозначения, а также играюший важную роль в дальнейшем изложении результат Зейбелла о существовании непрерывной, в смысле слабой сходимости, версии регулярного условного распределения на LCA-группе. B § 2 доказывается основной результат этой статьи (его формулировка приведена в [20]); последний параграф посвящен следствиям этого результата.

\section{§1. Основные определения и обозначения. Непрерывная версия условного распределения}

1.1. Некоторые сведения об LCA-группах. Пусть для $j=1$ и $j=2$ тройка $\mathbf{G}_{j} \equiv\left(G_{j},(+)_{j}, \mathfrak{U}_{j}\right)$ является LCA-группой с операцией сложения $(+)_{j}$ и топологией $\mathfrak{U}_{j}$ (в дальнейшем изложении вместо $(t)_{j}$ будет применяться и символ + , если это не вызовет недоразумений). Как известно (см., например, [4, с. 43]), на каждой LCA-группе существует мера Хаара $x$, являюшаяся инвариантной относительно групповой операции и единственной с точностью до мультипликативной константы. Произведение конечного числа LCA-групп с топологией произведения является LCA-группой (см., например, [21, предложение 16]) с мерой Хаара, равной произведению мер Хаара сомножителей.

Следуюшие группы являются «элементарными» LCA-группами. B группе действительных чисел $\mathbf{R}$ групповой операцией является обычное сложение, а топологией - обычная топология с совокупностью всех открытых интервалов в качестве своей базы; мера Хаара в R - меpa Лебега. Группа целых чисел $\mathbf{Z}$ является LCA-подгруппой группы $\mathbf{R}$; топология $\mathbf{Z}$ дискретна, а мерой Хаара является «считаюшая» мера, равная для каждого множества числу элементов $\mathbf{Z}$, в нем содержащихся. Множество Т всех комплексных чисел, равных по модулю единице, с умножением в качестве групповой операщии и с топологией, порожденной топологией комплексной плоскости, образует LCA-группу T; мерой Хаара для каждой «дуги» из $\mathbf{T}$ является длина этой дуги. Как и $\mathbf{Z}$, конечная дискретная абелева группа $\mathbf{F}$ имеет дискретную топологию и «считающую» меру в качестве меры Хаара.

Пространство $\mathbf{G}_{j}$ может рассматриваться и как группа $\left(G_{j},(+)_{j}\right)$, и как топологическое пространство $\left(G_{j}, \mathfrak{U}_{j}\right)$. Две группы $(G,+)$ и $\left(G^{\prime},+^{\prime}\right)$ называют алеебраически изоморфными, если существует отображение $f: G \rightarrow G^{\prime}$, являющееся взаимно однозначным («на») и сохраняющим групповую операцию, т.е. $f\left(g_{1}+g_{2}\right)=f\left(g_{1}\right)+^{\prime} f\left(g_{2}\right)$ для любых $g_{1}, g_{2} \in G$; два топологических пространства $(G, \mathfrak{U})$ и $\left(G^{\prime}, \mathfrak{U}^{\prime}\right)$ называют топологииески эквивалентными (или гомеоморфными), если существует отображение $f: G \rightarrow G^{\prime}$, являющееся взаимно однозначным («на») и непрерыв- 
ным, причем обратное отображение $f^{-1}$ также непрерывно. Две топологические (или непрерывные) группы $\mathbf{G}=(G,+, \mathfrak{U})$ и $\mathbf{G}^{\prime}=\left(G^{\prime},+^{\prime}, \mathfrak{U}^{\prime}\right)$ называют топологически изоморфными, если существует отображение $f: \mathbf{G} \rightarrow \mathbf{G}^{\prime}$, относительно которого эти группы являются алгебраически изоморфными и топологически эквивалентными.

Предположим, что $\mathbf{H}$ - замкнутая подгруппа LCA-rруппы G; тогда алгебраическая факторгруппа $\mathbf{G} / \mathbf{H}$, снабженная фактортопологией относительно канонического гомоморфизма $p$ (ставящего в соответствие каждому элементу $g \in G$ содержаший его элемент из множества $\mathbf{G} / \mathbf{H}$ классов смежности), образует LCA-группу - см. [21, предложения 10, 13 и 14].

Пусть $(G, \mathfrak{U})$ - топологическое пространство и $\mathfrak{B}$ - наименьшая $\sigma$-алгебра, содержащая $U$; при этом $\mathfrak{B}$ называется борелевской $\sigma$-алгеброй, ее элементы - борелевскими множествами, а пара $(G, \mathfrak{B})$ - борелевским пространством. Пусть $\left(G_{i}, \mathfrak{B}_{i}\right), i=1, \ldots, M$, - борелевские пространства; наименьшая $\sigma$-алгебра $\mathfrak{B}_{1} \times \cdots \times \mathfrak{B}_{M}$, содержашая все борелевские прямоугольники $B=B_{1} \times \cdots \times B_{M}, B_{i} \in \mathfrak{B}_{i}(i=$ $1, \ldots, M)$, называется произведением борелевских $\sigma$-алгебр $\mathfrak{B}_{1}, \ldots, \mathfrak{B}_{M}$, a пара $\left(G_{1} \times \cdots \times G_{M}, \mathfrak{B}_{1} \times \cdots \times \mathfrak{B}_{M}\right)$ называется произведением борелевских пространств $\left(G_{i}, \mathfrak{B}_{i}\right), i=1, \ldots, M$. Если $(G, \mathfrak{S})$ и $\left(G^{\prime}, \mathfrak{S}^{\prime}\right)-$ измеримые пространства, то функция $f: G \rightarrow G^{\prime}$ называется $\left(\mathfrak{S}^{\prime}, \mathfrak{S}\right)$ измеримой, если прообраз $f^{-1}[A]$ любого множества $A$ из $\mathfrak{S}^{\prime}$ принадлежит $\mathfrak{S}$. Если $\sigma$-алгебры $\mathfrak{S}$ и $\mathfrak{S}^{\prime}$ являются борелевскими, то $\left(\mathfrak{S}^{\prime}, \mathfrak{S}\right)$ измеримая функция $f$ называется борелевской; если, кроме того, $f$ является взаимно однозначным отображением («на») и обратное отображение является борелевским, то $f$ называется борелевским изоморфизмом борелевских пространств $(G, \mathfrak{S})$ и $\left(G^{\prime}, \mathfrak{S}^{\prime}\right)$. Борелевский изоморфизм двух одинаковых борелевских пространств называется борелевским автоморфизмом.

Если $\mathbf{G}=(G,+, \mathfrak{U})$ есть LCA-группа, то непрерывный гомоморфизм $\gamma: \mathbf{G} \rightarrow \mathbf{T}$ называется жепрерывным характером. На множестве $\mathbf{G}^{*}$ всех непрерывных характеров $\gamma$ можно ввести коммутативную операцию, определяемую формулой:

$$
\left(\gamma^{\prime}+\gamma^{\prime \prime}\right)(g)=\gamma^{\prime}(g) \gamma^{\prime \prime}(g)
$$

для всех $g \in \mathbf{G}$ и всех $\gamma^{\prime}, \gamma^{\prime \prime} \in \mathbf{G}^{*}$, и компактно-открытую топологию $\mathfrak{U}^{*}$, предбазу которой образуют все множества вида:

$$
M(K, V)=\left\{\gamma \in \mathbf{G}^{*}: \mathbf{G} \rightarrow \mathbf{T}, \gamma[K] \subseteq V\right\},
$$

где $K$ - произвольное компактное множество в $\mathbf{G}$ и $V$ - произвольное открытое множество в $\mathbf{T}$. Тройка $\mathbf{G}^{*} \equiv\left(G^{*},+, \mathfrak{U}^{*}\right)$ называется группой непрерывных характеров или группой, двойственной $\kappa \mathbf{G}$. Хорошо известно (см., например, [21, Предложение 29]), что $\mathbf{G}^{*}$ является LCAгруппой, так что существует $\boldsymbol{x}^{*}$ - мера Хаaра на $\mathbf{G}^{*}$. Для элементов последней группы наряду с $\gamma$ будет использоваться и обозначение $g^{*}$. 
Пусть $\mathbf{G}_{j}^{*}$ - группа, двойственная к $\mathbf{G}_{j}, j=1,2$; тогда произведение групп $\mathbf{G}^{*}=\mathbf{G}_{1}^{*} \times \mathbf{G}_{2}^{*}$ является (см. [21, теорема 13]) LCA-группой, двойственной к произведению групп $\mathbf{G}=\mathbf{G}_{1} \times \mathbf{G}_{2}$. Аниулятором $\mathbf{L}$ замкнутой подгруппы $\mathbf{H}$ LCA-группы $\mathbf{G}$ называется множество (снабженное индуцированной топологией) всех $\gamma \in \mathbf{G}^{*}$, таких, что $\gamma(h)=1$ для всех $h \in \mathbf{H}$; аннулятор представляет собой (см. [21, с. 74]) замкнутую подгруппу группы $\mathbf{G}^{*}$, так что $\mathrm{L}^{*}$ есть LCA-группа.

Для любого элемента $g$ из LCA-группы $\mathbf{G}$ определим функцию $g^{\prime}$ : $\mathbf{G}^{*} \rightarrow \mathbf{T}$ согласно формуле: $g^{\prime}(\gamma) \mapsto \gamma(g)$ для всех $\gamma \in \mathbf{G}^{*}$. Имеет место теорема двойственности Понтрягина (см. [23, теорема 52] и [21, теорема 23]): каноническое отображение $\alpha: \alpha(g) \mapsto g^{\prime}$ для всех $g \in \mathbf{G}$ является изоморфизмом топологической группы $\mathbf{G}$ на вторую двойственную групnу $\mathbf{G}^{* *}=\left(\mathbf{G}^{*}\right)^{*}$. $\mathrm{K}$ многочисленным следствиям этой теоремы относя тся, в частности, следуюшие: а) группа $\mathbf{G}^{*}$ компактна, если группа $\mathbf{G}$ дискретна; 6 ) группа $\mathbf{G}^{*}$ дискретна, если $\mathbf{G}$ компактна; в) из первых двух утверждений следует, что для всех натуральных значений $n \geqslant 1$ топологические группы $\mathbf{Z}^{n}$ и $\mathrm{T}^{n}$ являются двойственными по отношению друг к другу; г) если $\mathbf{H}-$ замкнутая подгруппа LCA-группы $\mathbf{G}$, то топологические группы $\mathrm{L}$ и $\mathbf{G}^{*} / \mathrm{L}$ топологически изоморфны $[21$, теорема 27]) группам $(\mathbf{G} / \mathbf{H})^{*}$ и $\mathbf{H}^{*}$ соответственно.

1.2. Условная характеристическая функция и регулярное условное распределение. Если $X$ - случайная величина, принимающая значения в LCA-группе $\mathbf{G}$, и $\mathbf{P} X^{-1}$ - ее распределение, то характеристическая функиия (х.ф.) $\varphi_{X}$ величины $X$ определяется преобразованием Фурье

$$
\varphi_{X}(\gamma)=\int_{G} \gamma(g) d \mathbf{P} X^{-1}(g)
$$

для всех $\gamma \in \mathbf{G}^{*}$. Совместная характеристическая функция $\varphi_{X, Y}$ случайных величин $X$ и $Y$, заданных на вероятностном пространстве $(\Omega, \mathfrak{S}, \mathbf{P})$ и принимаюши значения в LCA-группах $\mathbf{G}_{1}$ и $\mathbf{G}_{2}$ соответственно, определяется формулой:

$$
\varphi_{X, Y}(\gamma)=\int_{G_{1} \times G_{2}} \prod_{j=1}^{2} \gamma_{j}\left(g_{j}\right) d \mathrm{P}(X, Y)^{-1}(g)
$$

для всех $\gamma \equiv\left(\gamma_{1}, \gamma_{2}\right) \in \mathbf{G}_{1}^{*} \times \mathbf{G}_{2}^{*}$, где $\mathbf{P}(X, Y)^{-1}$ - совместное распределение случайных величин $X, Y$ и $g=\left(g_{1}, g_{2}\right)$.

Функция $\mathbf{Q}_{X \mid Y}(\cdot, \cdot)$ называется регулярным условным распределением случайной величины $X$ при заданной случайной величине $Y$, если для каждого значения $y \in G_{2}$ функция $\mathbf{Q}_{X \mid Y}(y, \cdot)$ является вероятностным распределением на $\mathfrak{S}_{1}$ и для каждого значения $B \in \mathfrak{S}_{1}$ функция $\mathbf{Q}_{X \mid Y}(\cdot, B)$ является вариантом условной вероятности

$$
\mathbf{P}_{X \mid Y}(\{\omega \in \Omega: X(\omega) \in B\} \mid \cdot)
$$


при заданной случайной величине $Y$. В этом случае для любой действительной случайной величины $h$, определенной на вероятностном пространстве $(\Omega, \mathfrak{S}, \mathbf{P})$ и имеющей конечное математическое ожидание, почти наверное (относительно $\mathrm{P}^{-1}$ ) справедливо равенство:

$$
\int_{G_{1}} h(x) \mathbf{Q}_{X \mid Y}(y, d x)=\mathbf{E}(h \mid Y=y),
$$

где правая часть является условным математическим ожиданием случайной величины $h$ при заданном значении $y$ случайной величины $Y$. Согласно (1.4), условная характеристическая функиия $\varphi_{X \mid Y}(\cdot \mid y)$ случайной величины $X$ при условии $Y=y$ определяется формулой:

$$
\varphi_{X \mid Y}\left(\gamma_{1} \mid Y=y\right)=\int_{G_{1}} \gamma_{1}\left(g_{1}\right) \mathbf{P}_{X \mid Y}\left(d g_{1} \mid Y=y\right)
$$

для всех $\gamma_{1} \in G_{1}^{*}$ и почти всех (относительно $\mathrm{P} Y^{-1}$ ) $y \in G_{2}$. Если LCAгруппы $\mathbf{G}_{1}$ и $\mathbf{G}_{2}$ являются полными сепарабельными метрическими пространствами, то существует [22, утверждение 46.3] регулярное условное распределение $\mathbf{Q}_{X \mid Y}(\cdot, \cdot)$. Условное распределение $\mathbf{Q}_{X \mid Y}(\cdot, \cdot)$ называется непрерывным в смысле слабой сходимости на множестве $V$, если для любых $y, y_{0} \in V$

$$
\mathbf{Q}_{X \mid Y}(y, \cdot) \Longrightarrow \mathbf{Q}_{X \mid Y}\left(y_{0}, \cdot\right) \text { при } y \rightarrow y_{0},
$$

где символ $\Rightarrow$ обозначает слабую сходимость распределений.

1.3. Основные определения. Предположим, что случайные величины $w_{1}, \ldots, w_{N}$ и независимые в совокупности случайные величины $\theta_{1}, \ldots, \theta_{N}$, определенные на вероятностных пространствах $\left(\Omega_{1}, \mathfrak{S}_{1}, \mathbf{P}_{1}\right)$ и $\left(\Omega_{2}, \mathfrak{S}_{2}, \mathbf{P}_{2}\right)$ соответственно, принимают значения в LCA-группе $\mathbf{G}_{2}=$ $\left(G_{2},+, \mathfrak{U}_{2}\right)$, и пусть

$$
w(N)=\left(w_{1}, \ldots, w_{N}\right) \text { и } \theta(N)=\left(\theta_{1}, \ldots, \theta_{N}\right) .
$$

Предположим, что на произведении множеств $G_{2}^{l+1}$ заданы функции $f_{k}$, $k=1, \ldots, N$, принимающие значения в LCA-группе $\mathbf{G}_{1}=\left(G_{1},+, \mathfrak{U}_{1}\right)$; введем функцию

$$
f:\left(g_{2}^{(1)}, \ldots, g_{2}^{(N)}\right) \mapsto \sum_{k=1}^{N} f_{k}\left(g_{2}^{(k)}, \ldots, g_{2}^{(k+1)}\right)
$$

где

$$
g_{2}^{(k)} \in G_{2}, \quad k=1, \ldots, N, \quad g_{2}^{(k+N)}=g_{2}^{(k)}, \quad k=1, \ldots, l .
$$

Учитывая соотношения (1.8), рассмотрим сумму

$$
\zeta_{N}=\sum_{k=1}^{N} f_{k}\left(w_{k}, \ldots, w_{k+l}\right)
$$

и введем «сопутствующие» случайной величине $\zeta_{N}$ суммы:

$$
\xi_{N}=\sum_{k=1}^{N} f_{k}\left(\theta_{k}, \ldots, \theta_{k+l}\right), \quad \eta_{N}=\sum_{k=1}^{N} \theta_{k} .
$$


Пусть $\mathfrak{B}_{j}$ - наименьшая $\sigma$-алгебра, содержащая топологию $\mathfrak{U}_{j}$, $(j=1,2)$.

Отметим следующие обстоятельства. Во-первых, если точка «а» не является атомом распределения суммы $\eta_{N}$, то при условии фиксации последней в «a» выбор версии условного распределения вектора $\theta(N)$ неоднозначен, так что в этой ситуации случайные величины $w_{1}, \ldots, w_{N}$ не определены. Указанная неопределенность исчезает, если потребовать существование версии упомянутого условного распределения, непрерывной (в смысле слабой сходимости) в какой-нибудь окрестности точки « $a$ » относительно некоторой топологии; в свою очередь, последнее требование эквивалентно существованию непрерывной в некоторой окрестности точки «a» версии условной х.Ф. Во-вторых, нам интересен тот случай, при котором распределение случайной величины (1.9) совпадает с условным распределением суммы $\zeta_{N}$ при условии $\eta_{N}=a$. Поэтому мы предполагаем существование версии этого условного распределения, непрерывной (в смысле слабой сходимости) в какой-нибудь окрестности точки «a», и то, что эта версия совпадает с версией, индуцированной непрерывным вариантом ранее упомянутого условното распределения. В-третьих, формулы (1.9) и (1.10) основаны на коммутативных операциях, и естественное требование (в частности, при округлении значений действительных функций $f_{k}$ от действительных аргументов) к таким операциям состоит в их непрерывности относительно некоторых топологий. Если, например, $G_{1}=\mathbf{R}$ и $G_{2}=\mathbf{Z}$, то в качестве топологий выбираются «обычная», как правило, и дискретная топологии соответственно. Следующее определение принадлежит автору [17]-[19], [38].

O п р е д е л е н и е 1. Величина $\zeta_{N}$, определенная формулой (1.9), называется $l$-разделимой статистикой (l-PC, для краткости), если выполняются следуюшие условия.

1) Функпия $f$ является $\left(\mathfrak{B}_{1}, \mathfrak{B}_{2}^{N}\right)$-измеримой.

2) Сушествуют независимые в совокупности случайные величины $\theta_{1}, \ldots, \theta_{N}$, определенные на некотором вероятностном пространстве $\left(\Omega_{2}, \mathfrak{S}_{2}, \mathbf{P}_{2}\right)$, и элемент $a \in G_{2}$, такие, что: а) суммы (1.10) являются случайными величинами; б) распределение $\mathrm{P}_{1}(w(N))^{-1}$ вектора $w(N)$ удовлетворяет соотношению

$$
\mathbf{P}_{\mathbf{1}}(w(N))^{-1}(B)=\hat{\mathbf{Q}}_{\theta(N) \mid \eta_{N}}(a, B)
$$

для всех $B \in \mathfrak{B}_{2}^{N}$, где $\widehat{\mathbf{Q}}_{\theta(N) \mid \eta_{N}}(\cdot, \cdot)$ - непрерывная в смысле слабой сходимости, в окрестности $V$ точки «a» версия регулярного условного распределения $\mathbf{Q}_{\theta(N) \mid \eta_{N}}(\cdot, \cdot)$ случайного вектора $\theta(N)$ при заданной случайной величине $\eta_{N} ;$ в) версия $\widehat{\mathbf{Q}}_{\xi_{N} \mid \eta_{N}}(\cdot, \cdot)$ условного распределения случайной величины $\xi_{N}$ при заданной случайной величине $\eta_{N}$, определяемая для всех $y \in G_{2}$ и всех $A \in \mathfrak{B}_{1}$ формулой

$$
\widehat{\mathbf{Q}}_{\xi_{N} \mid \eta_{N}}(y, A)=\widehat{\mathbf{Q}}_{\theta(N) \mid \eta_{N}}\left(y, f^{-1}[A]\right),
$$


регулярна и непрерывна, в смысле слабой сходимости, в окрестности $V^{*}$ «точки» $a$.

В работе.[38] приведены широкие достаточные условия существования разделимых статистик. Согласно части 1) определения 1 , сумма $\zeta_{N}$ является (см. [22, упражнение 22.11]) случайной величиной, и в силу условия 2.а) и формул (1.11) и (1.12) для любого $A \in \mathfrak{B}_{1}$ имеем:

$$
\begin{aligned}
\mathbf{P}_{1} \zeta_{N}^{-1}(A) & =\widehat{\mathbf{Q}}_{\theta(N) \mid \eta_{N}}\left(a, f^{-1}[A]\right) \\
& =\widehat{\mathbf{P}}_{2}\left(\left\{\omega_{2} \in \Omega_{2}: f\left(\theta(N)\left(\omega_{2}\right)\right) \in A\right\} \mid \eta_{N}=a\right)
\end{aligned}
$$

где $\mathbf{P}_{1} \zeta_{N}^{-1}$ - распределение случайной величины $\zeta_{N}$ и $\widehat{\mathbf{P}}_{2}(\cdot, \cdot)-$ соответствующая версия условного распределения на $\sigma$-алгебре $\mathfrak{S}_{\xi_{N}} \subset \mathfrak{S}_{2}$, индуцируемой случайной величиной $\zeta_{N}$, при заданной случайной величине $\eta_{N}$.

Замкнутое множество $C_{\mu}$ (или $\operatorname{supp} \mu$ ) называется носителем вероятностной меры $\mu$ (см., например, $\left[42\right.$, с. 28-29]), если $\mu\left(C_{\mu}\right)=1$ и для любого замкнутого множества $C$ условие $\mu(C)=1$ влечет соотношение $C_{\mu} \subset C$. Следуюшие три определения введены [19], [38] автором.

О п р ед е л е н и е 2. Набор

$$
\left(N, a, \mathbf{G}_{2},\left\{\mathbf{P}_{2} \theta_{k}^{-1} ; k=1, \ldots, N\right\}, \mathbf{G}_{1}\right)
$$

называется схемой разделимых статистик (схемой $P C$, для краткости), если $\mathbf{G}_{2}$ есть наименьшая LCA-группа, порожденная множеством носителей распределений $\mathbf{P}_{2} \theta_{k}^{-1}$ случайных величин $\theta_{k}, k=1, \ldots, N$, и если $\mathbf{G}_{1}$ - наименьшая LCA-группа, порожденная носителем распределения $\mathbf{P}_{1} \xi^{-1}$ случайной величины $\boldsymbol{\xi}$.

Набор (1.14) является основой для классификации [19] РС; каждая $l$-РC определяется соответствующей схемой $\mathrm{PC}$ и набором функций $f_{k}$, $k=1, \ldots, N$.

O п р е д е л н и е 3 . Схема разделимых статистик называется симметрической, если распределения $\mathrm{P}_{2} \theta_{k}^{-1}$ случайных величин $\theta_{k}, k=$ $1, \ldots, N$, совпадают; в противном случае схема разделимых статистик называется несимметрической.

О п р е д е л е н и е 4. l-разделимая статистика называется симметрической, если соответствующая ей схема разделимых статистик является симметрической и если все функции $f_{k}, k=1, \ldots, N$, совпадают; в противном случае $l$-разделимая статистика называется несимметричеcкой.

1.4. Непрерывная версия регулярного условного распределения. Ее существование для случайной величины $X$ при заданной случайной величине $Y$ связано с интегральным представлением для условной х.ф. случайной величины $X$ при заданной случайной величине $Y$. Для случаев, когда величины $X$ и $Y$ являются действительнозначными или векторными с действительными компонентами, такое представление получено Уикселлом [47], Бартлеттом [32], Йе [48], Стэком [45], а для 
обшего случая, когда $X$ и $Y$ принимают значения в LCA-группах (скажем, $\mathbf{G}_{1}$ и $\mathbf{G}_{2}$ соответственно), - Зейбеллом [49]. Предположим, что в последнем случае выполняются условия: 1) LCA-группы $\mathbf{G}_{1}$ и $\mathbf{G}_{2}$ являются полными сепарабельными метрическими пространствами; 2) $\mathbf{G}_{2}$ является наименьшей LCA-группой, порожденной носителем распределения случайной величины $Y$;3) существует не зависяпая от $\gamma_{1} \in G_{1}^{*}$ неотрицательная борелевская функция $\Delta \equiv \Delta\left(\gamma_{2}\right)$ такая, что совместная х.ф. (1.3) для всех $\gamma \equiv\left(\gamma_{1}, \gamma_{2}\right) \in G_{1}^{*} \times G_{2}^{*}$ удовлетворяет неравенству $\left|\varphi_{X, Y}(\gamma)\right| \leqslant \Delta\left(\gamma_{2}\right)$, причем $\int_{G_{2}^{*}} \Delta\left(\gamma_{2}\right) d x_{2}^{*}\left(\gamma_{2}\right)<+\infty$.

В частности, из этих условий и теоремы обращения Фурье вытекает существование непрерывной в топологии $\mathfrak{U}_{2}$ ограниченной плотности (по отношению к мере Хаара $\varkappa_{2}$ на $\mathbf{G}_{2}$ ) распределения случайной величины $Y$, причем для всех $y \in G_{2}$ она определяется формулой

$$
\frac{d \mathrm{P} Y^{-1}}{d \varkappa_{2}}(y)=C \int_{G_{2}^{*}} \varphi_{X, Y}\left(e_{1}^{*}, \gamma_{2}\right) \gamma_{2}(-y) d \varkappa_{2}^{*}\left(\gamma_{2}\right)
$$

в (1.15) $e_{1}^{*}$ - нейтральный элемент группы $\mathbf{G}_{1}^{*}$, а константа $C$ может быть включена в меру Хаара $\varkappa_{2}^{*}$. Далее, при выполнении условий 1)-3), согласно Зейбеллу ([49, теоремы 3.1 и 3.2$])$, для всех $y \in V_{0}$,

$$
V_{0}=\left\{g_{2} \in G_{2}: \frac{d \mathrm{P} Y^{-1}}{d \varkappa_{2}}\left(g_{2}\right)>0\right\},
$$

и всех $\gamma_{1} \in G_{1}^{*}$ формула

$$
\begin{aligned}
\hat{\varphi}_{X \mid Y}\left(\gamma_{1} \mid Y=y\right)= & \int_{G_{2}^{*}} \varphi_{X, Y}\left(\gamma_{1}, \gamma_{2}\right) \gamma_{2}(-y) d \varkappa_{2}^{*}\left(\gamma_{2}\right) \\
& \times\left\{\int_{G_{2}^{*}} \varphi_{X, Y}\left(e_{1}^{*}, \gamma_{2}\right) \gamma_{2}(-y) d \varkappa_{2}^{*}\left(\gamma_{2}\right)\right\}^{-1}
\end{aligned}
$$

определяет версию условной х.ф. $\varphi_{X \mid Y}(\cdot, \cdot)$ случайной величины $X$ при заданной случайной величине $Y$, и, кроме того, функция $\widehat{\mathbf{Q}}_{X \mid Y}(\cdot, \cdot)$, однозначно определяемая для всех $\gamma_{1} \in G_{1}^{*}$ и всех $y \in V_{0}$ формулой

$$
\widehat{\varphi}_{X \mid Y}\left(\gamma_{1} \mid Y=y\right)=\int_{G_{1}} \gamma_{1}\left(g_{1}\right) \widehat{\mathbf{Q}}_{X \mid Y}\left(y, d g_{1}\right),
$$

является непрерывной, в смысле слабой сходимости, версией регулярного условного распределения случайной величины $X$ при заданной случайной величине $Y$ (отметим, что в силу непрерывности плотности (1.15) множество (1.16) является открытым в топологии $\mathfrak{U}_{2}$ и что для второго множителя, стоящего в правой части (1.17), можно воспользоваться представлением (1.15)). Этот результат доказан в [49] для случая $G_{1}=G_{2} ;$ в ситуации $G_{1} \neq G_{2}$ доказательство проводится аналогично. Заметим, что правая часть (1.17) не зависит от выбора варианта меры Xaapa $\boldsymbol{x}_{2}^{*}$. 


\section{§ 2. Основная предельная теорема}

2.1. Формулировка основного результата. Здесь, в отличие от предыдущего, мы будем иметь дело с последовательностями l-PC:

$$
\zeta_{N}=\sum_{k=1}^{N} f_{N k}\left(w_{N k}, \ldots, w_{N, k+1}\right), \quad N \geqslant 2,
$$

где суммирование, ках и рағее, происходит по модулю $N$ и значение $l$, вообще говоря, может зависеть от $N$. При этом, не нарушая общности, можно считать, что при каждом $N \geqslant 2$ случайные величины $w_{N 1}, \ldots, w_{N N}$ и соответствуюшие им независимые случайные величины $\theta_{N 1}, \ldots, \theta_{N N}$ определены на не зависящих от $N$ вероятностных пространствах $\left(\Omega_{1}, \mathfrak{S}_{1}, \mathbf{P}_{1}\right)$ и $\left(\Omega_{2}, \mathfrak{S}_{2}, \mathbf{P}_{2}\right)$ соответственно. Вместо обозначений $V$ и $a$, упомянутых определением 1 , будем употреблять $V_{N}$ и $a_{N}^{0}$, а вместо (1.6) и (1.10) будем пользоваться обозначениями:

$$
\begin{gathered}
w(N)=\left(w_{N 1}, \ldots, w_{N N}\right), \quad \theta(N)=\left(\theta_{N 1}, \ldots, \theta_{N N}\right), \\
\xi_{N}=\sum_{k=1}^{N} f_{N k}\left(\theta_{N k}, \ldots, \theta_{N, k+1}\right), \quad \eta_{N}=\sum_{k=1}^{N} \theta_{N k} .
\end{gathered}
$$

Пусть при каждом $N \geqslant 2$ множество значений случайных величин $f_{N k}\left(\theta_{N k}\right)$ и множество значений случайных величин $\theta_{N k}, k=1, \ldots, N$, принадлежит, соответственно, замкнутым подгруппам $G_{N 1}$ и $G_{N 2}$ подходяших LCA-групп $\mathbf{G}_{1} \equiv\left(G_{1},+, \mathfrak{U}_{1}\right)$ и $\mathbf{G}_{2} \equiv\left(G_{2},+, \mathfrak{U}_{2}\right)$. Для каждого $N \geqslant 2$ и $j=1,2$ подгруппы $\mathbf{G}_{N j} \equiv\left(G_{N j},+, \mathfrak{U}_{N j}\right)$, где $\mathfrak{U}_{N j}=\mathfrak{U}_{j} \cap G_{N j}$, сами являются LCA-группами (см., например, предложение 4 из [21] и 1-й абзац на с. 196 в [7]). Для $j=1,2$ обозначим через $\mathfrak{B}_{N j}$ наименьшую $\sigma$-алгебру, содержащую топологию $\mathfrak{U}_{N j}$, а через $\mathbf{G}_{j}^{*}$ и $\mathbf{G}_{N j}^{*}-$ групшы, двойственные к группам $\mathbf{G}_{j}$ и $\mathbf{G}_{N j}$ соответственно. Пусть $\mathbf{L}_{N j}-$ аннулятор подгруппы $\mathbf{G}_{N j}$, и пусть $p_{N j}: \mathbf{G}_{j}^{*} \rightarrow \mathbf{G}_{j}^{*} / \mathbf{L}_{N j}-$ канонический гомоморфизм, ставящий в соответствие каждому элементу из $\mathbf{G}_{j}$ содержащий его класс смежности. Согласно утверждению г) пункта 1.1 , для $j=1,2$ существуют топологические изоморфизмы $v_{N j}: \mathbf{G}_{j}^{*} / \mathbf{L}_{N j} \rightarrow \mathbf{G}_{N j}^{*}$, $N \geqslant 2$. Обозначим через $\varphi_{N}$ совместную х.ф. величин $\xi_{N}$ и $\eta_{N}$.

Теорема. Предположим, что выполняются следуючие условия, первые четыре из которых имеют место при всех $N \geqslant 2$ :

а) для каждого $k=1, \ldots, N$ бункиии $f_{N k}$ являются $\left(\mathfrak{B}_{N 1}, \mathfrak{B}_{N 2}^{l+1}\right)$ измеримыми;

б) величина (2.1) явлдется l-разделимой статистикой в смысле определения 1 ;

в) еруппа $\mathbf{G}_{N 2}$ порождена носителем распределения случайной өеличины $\eta_{N}$, определяемой формулой (2.3);

г) LCA-группы $\mathbf{G}_{1} \quad$ u $\mathbf{G}_{2}$ яөляются полными сепарабельными метрическими пространствами; 
д) для любых не зависящих от $N$ элементов $\gamma_{j} \in \mathbf{G}_{j}^{*}(j=1,2)$ последовательность значений $\varphi_{N}\left(v_{N 1}\left(p_{N 1}\left(\gamma_{1}\right)\right), v_{N 2}\left(p_{N 2}\left(\gamma_{2}\right)\right)\right)$ nрu $N \rightarrow \infty$ сходится $\boldsymbol{x}$ значению $\varphi(\gamma), \gamma=\left(\gamma_{1}, \gamma_{2}\right)$, некоторой функиии $\varphi$, непрерывной в нейтральном элементе $\left(e_{1}^{*}, e_{2}^{*}\right)$ произведения групп $\mathbf{G}_{1}^{*} \times \mathbf{G}_{2}^{*}$;

е) существует неотричательная борелевская функиия $\Delta$, определенная на $\mathrm{G}_{2}^{*}$, и натуральное число $N_{1} \geqslant 2$, такие, что при всех значениях $\gamma_{j} \in \mathbf{G}_{j}^{*}, j=1,2$, выполняютося неравенства

$$
\left|\varphi_{N}\left(v_{N 1}\left(p_{N 1}\left(\gamma_{1}\right)\right), v_{N 2}\left(p_{N 2}\left(\gamma_{2}\right)\right)\right)\right| \leqslant \Delta\left(\gamma_{2}\right), \quad N \geqslant N_{1},
$$

причем функиия $\Delta$ является интегрируемой на $\mathrm{G}_{2}^{*}$ по мере Хаара $\varkappa_{2}^{*}$. Tогдa:

1) функция $\varphi \equiv \varphi_{\xi, \eta}$ является совместной х.ф. некоторых слуиайных величин $\xi, \eta$ со значениями в $\mathrm{G}_{1}$ u $\mathrm{G}_{2}$ соответственно;

2) в асuмnmomuxe

$$
N \rightarrow \infty, \quad a_{N}^{0} \in \widetilde{V}_{N} \rightarrow a^{0} \in G_{2},
$$

где $\widetilde{V}_{N}=\left\{g_{N 2} \in G_{N 2}:\left(d \mathbf{P}_{2} \eta_{N}^{-1} / d \varkappa_{N 2}\right)\left(g_{N 2}\right)>0\right\}$, и при справедливости yсловия

$$
\frac{d \mathrm{P}_{2} \eta^{-1}}{d \varkappa_{2}}\left(a^{0}\right) \neq 0
$$

последовательность распределений случайной величины $\zeta_{N}$ слабо сходится к версии регулярного условного распределения $\mathbf{P}_{\xi \mid \eta}$, непрерывной, в смысле слабой сходимости, на множестве

$$
V=\left\{v \in G_{2}: \frac{d \mathrm{P}_{2} \eta^{-1}}{d \varkappa_{2}}>0\right\}
$$

u определяемой правой частью нижеследующей формулы (2.16). Здесь $\left.d \mathbf{P}_{2} \eta_{N}^{-1} / d \varkappa_{N 2}\right), d \mathbf{P}_{2} \eta^{-1} / d \varkappa_{2}$ - ограниченные и непрерывные (на $\widetilde{V}_{N} u V$ соответственно) версии плотностей распределения случайных величин $\eta_{N}, \eta$ по отночения $к$ мерам Хаара $\varkappa_{N 2}, \varkappa_{2}$ иа $\mathbf{G}_{N 2} u \mathbf{G}_{2}$ coотөетственно.

2.2. Доказательство теоремы. Доказательство проведем в несколько этапов.

1) Согласно условию г) и в силу своей замкнутости группы $\mathbf{G}_{N j}$ $(N \geqslant 2 ; j=1,2)$, а также их всевозможные конечные произведения, являются полными сепарабельными пространствами. Отсюда, с учетом условия а), следует, в частности, соответствующая измеримость сумм (2.1) и (2.3). При этом существуют (см. [42, с. 27 , теорема 2.1]) и носители распределений, упомянутые условием в).

2) Группы непрерывных характеров $\mathbf{G}_{1}^{*}$ и $\mathbf{G}_{2}^{*}$, а также их произведение, являются LCA-группами (согласно теоремам 10 и 13 из [21]) и топологическими пространствами со второй аксиомой счетности (из-за наличия такого свойства у топологических пространств $\mathbf{G}_{1}$ и $\mathbf{G}_{2}$ и в силу теоремы 57 из [23]). Последнее обстоятельство влечет, в частности, 
$\sigma$-конечность мер Хаара этих групा (см. [22, утверждение 54.2]), что, в свою очередь, позволяет (см. [22, утверждение 29.9]) воспользоваться указанными ниже заменами переменных под знаком интеграла Лебега.

3) Совместная х.ф. $\varphi_{N}$ случайных величин $\xi_{N}$ и $\eta_{N}$ определена на произведении LCA-групп $\mathbf{G}_{N 1}^{*} \times \mathbf{G}_{N 2}^{*}$, а упомянутая условием д) предельная функция $\varphi-$ на произведении LCA-групा $\mathbf{G}_{1}^{*} \times \mathbf{G}_{2}^{*}$. Чтобы воспользоваться методом х.ф. в случае LCA-групп (см. [42, теоремы 3.1, $3.3]$, и $\left[24\right.$, с. 22-23]), мы с помошью композиций $v_{N j} \odot p_{N j}(j=1,2)$ определили согласно условию д) х.ф. $\varphi_{N}$ на всем пространстве $\mathrm{G}_{1}^{*} \times \mathrm{G}_{2}^{*}$.

Согласно одному из следствий теоремы двойственности Понтрягина (см. [21, следствие из теоремы 27]), каждый непрерывный характер $\gamma_{N j} \in \mathbf{G}_{N j}^{*}$ является сужением на $\mathbf{G}_{N j}$ некоторого непрерывного характера $\gamma_{j} \in \mathbf{G}_{j}^{*}(j=1,2)$, так что имеет место соотношение:

$$
\gamma_{N j}\left(g_{N j}\right)=\gamma_{j}^{\prime}\left(g_{N j}\right)
$$

для всех $g_{N j} \in G_{N j}$ и любого характера $\gamma_{j}^{\prime}$ из смежного класса $\gamma_{j}+L_{N j}$ факторгруппы $\mathbf{G}_{j}^{*} / \mathbf{L}_{N j}$, к которому принадлежит характер $\gamma_{j}$. Действительно, если $\gamma_{j}^{\prime}=\gamma_{j}+\lambda_{N j}$ с $\lambda_{N j} \in L_{N j}$, то $\gamma_{j}^{\prime}\left(g_{N j}\right)=\left(\gamma_{j}+\lambda_{N j}\right)\left(g_{N j}\right)=$ $\gamma_{j}\left(g_{N j}\right)+\lambda_{N j}\left(g_{N j}\right)=\gamma_{j}\left(g_{N j}\right)$, так как, согласно определению аннулятора, значение $\lambda_{N j}\left(g_{N j}\right)$ равно нейтральному элементу групाты $\mathbf{T}$ для всех $g_{N j} \in G_{N j}$ и всех $\lambda_{N j} \in L_{N j}$. С другой стороны, сужение на подтруппу $\mathbf{G}_{N j}$ произвольного непрерывного характера является гомоморфным и непрерывным (в относительной топологии), так что каждому смежному классу $B_{N j}$ из факторгруппы $\mathbf{G}_{j}^{*} / \mathbf{L}_{N j}$ соответствует непрерывный характер $\gamma_{N j}^{0}$ группы $\mathbf{G}_{N j}$. Это соответствие и является (см., например, теорему 27 из [21] и ее доказательство) упомянутым выше топологическим изоморфизмом $v_{N j}$. Очевидно, что значения $\varphi_{N}\left(v_{N 1}\left(p_{N 1}\left(\gamma_{1}^{\prime}\right)\right), v_{N 2}\left(p_{N 2}\left(\gamma_{2}^{\prime}\right)\right)\right)$ одинаковы для всех $\gamma^{\prime} \equiv\left(\gamma_{1}^{\prime}, \gamma_{2}^{\prime}\right)$ из произведения смежных классов $B_{N 1} \times B_{N 2}$, где $B_{N j}-$ смежный класс, содержащий элемент $\gamma_{j}(j=1,2)$.

Указанное «продолжение» х.ф. $\varphi_{N}$ соответствует продолжению совместного распределения случайных величин $\xi_{N}$ и $\eta_{N}$ с произведения $\mathbf{G}_{N 1} \times \mathbf{G}_{N 2}$ на произведение $\mathbf{G}_{1} \times \mathbf{G}_{2}$, задаваемому значением

$$
\mathbf{P}\left(\xi_{N}, \eta_{N}\right)^{-1}\left(B \cap G_{N 1} \times G_{N 2}\right)
$$

для любого борелевского множества $B$ из $\mathbf{G}_{1} \times \mathrm{G}_{2}$. Так как: а) имеет место взаимнооднозначное соответствие между мерами и х.ф., б) борелевская $\sigma$-алгебра произведения пространств $\mathbf{G}_{N 1} \times \mathbf{G}_{N 2}$ совпадает с произведением $\mathfrak{B}_{N 1} \times \mathfrak{B}_{N 2}$ (в силу наличия второй аксиомы счетности у пространств $\left.\mathbf{G}_{N 1}, \mathbf{G}_{N 2}\right)$, в) отображения $v_{N j}$ и $p_{N j}(j=1,2)$ непрерывны, то существует случайный вектор $\left(\xi_{N}^{\prime}, \eta_{N}^{\prime}\right)$ со значениями в $\mathbf{G}_{1} \times \mathbf{G}_{2}$, для которого функция со значениями $\varphi_{N}\left(v_{N 1}\left(p_{N 1}\left(\gamma_{1}\right)\right), v_{N 2}\left(p_{N 2}\left(\gamma_{2}\right)\right)\right)$, $\left(\gamma_{1}, \gamma_{2}\right) \in \mathbf{G}_{1} \times \mathbf{G}_{2}$, является х.ф. В силу условия д), наличия второй аксиомы счетности у пространств $\mathbf{G}_{1}, \mathbf{G}_{2}$ и метода х.ф. получим утверждение 1). 
4) Мера Хаара $\varkappa_{2}^{*}$ определена на борелевских множествах пространства $\mathbf{G}_{2}^{*}$, обладающего (см. этап 2)) второй аксиомой счетности; поэтому, в силу единственности, с точностью до мультипликативной константы, инвариантной меры на LCA-группе и согласно утверждению 54.2 из [22], $\varkappa_{2}^{*}$ является $\sigma$-конечной мерой.

Покажем, что мера $x_{2}^{*} p_{N 2}^{-1}$ на $\mathbf{G}_{2}^{*} / \mathbf{L}_{N 2}$, индуцируемая отображением $p_{N 2}$ из меры $x_{2}^{*}$ на $\mathrm{G}_{2}^{*}$, является мерой Хаара и, кроме того, $\sigma$-конечной мерой. Пусть $B_{N 2}^{0}$ и $B_{N 2}=\left\{B_{N 2}^{t}, t \in M\right\}-$ соответственно, произвольные элемент и борелевское множество из пространства $\mathbf{G}_{2}^{*} / \mathbf{L}_{N 2}$. Пусть $\gamma_{N}^{0}$ и $\gamma_{N}^{t}$ - «представители» смежных классов $B_{N 2}^{0}$ и $B_{N 2}^{t}$ соответственно, а $\mathbf{C}_{N}=\left\{\gamma_{N}^{t}, t \in M\right\}$. Согласно определению операции сложения в групте $\mathbf{G}_{2}^{*} / \mathbf{L}_{N 2}$ имеем:

$$
\begin{aligned}
\varkappa_{2}^{*} p_{N 2}^{-1}\left(B_{N 2}+{ }^{\prime} B_{N 2}^{0}\right) & =x_{2}^{*} p_{N 2}^{-1}\left(\left\{p_{N 2}\left(\gamma_{N}^{t}\right), t \in M\right\}+{ }^{\prime} p_{N 2}\left(\gamma_{N}^{0}\right)\right) \\
& =x_{2}^{*} p_{N 2}^{-1}\left(\left\{p_{N 2}\left(\gamma_{N}^{t}\right), t \in M\right\}\right)=x_{2}^{*} p_{N 2}^{-1}\left(B_{N 2}\right),
\end{aligned}
$$

так как $\varkappa_{2}^{*}\left(\mathbf{C}_{N}+\gamma_{N}^{0}\right)=\boldsymbol{x}_{2}^{*}\left(\mathbf{C}_{N}\right)$ в силу инвариантности меры $\boldsymbol{x}_{2}^{*}$. Следовательно, $x_{2}^{*} p_{N 2}^{-1}$ - мера Хаара на пространстве $\mathbf{G}_{2}^{*} / \mathbf{L}_{N 2}$, обладающем (см. утверждение $\mathrm{G}$ на с. 118 в [23]) второй аксиомой счетности, и она см. предыдуший абзац - является $\sigma$-конечной.

Так как $v_{N 2}$ - топологический изоморфизм, то для произвольных элемента $\gamma_{N 2}^{0}$ и борелевского множества $D_{N 2}$ из $\mathrm{G}_{N 2}^{*}$ с учетом предыдущего абзаца получим:

$$
\begin{aligned}
\varkappa_{2}^{*} p_{N 2}^{-1}\left(v_{N 2}^{-1}\left(D_{N 2}+{ }^{\prime} \gamma_{N 2}^{0}\right)\right) & =\varkappa_{2}^{*} p_{N 2}^{-1}\left(v_{N 2}^{-1}\left(D_{N 2}\right)+v_{N 2}^{-1}\left(\gamma_{N 2}^{0}\right)\right) \\
& =\varkappa_{2}^{*} p_{N 2}^{-1}\left(v_{N 2}^{-1}\left(D_{N 2}\right)\right) .
\end{aligned}
$$

Поэтому мера $x_{N 2}^{*}$ на $\mathrm{G}_{N 2}^{*}$, индуцируемая отображением $v_{N 2}$ из меры $\varkappa_{2}^{*} p_{N 2}^{-1}$ на $\mathbf{G}_{N 2}^{*} / \mathbf{L}_{N 2}$, является $\sigma$-конечной мерой Хаара.

5) На основании $\sigma$-конечности мер Хаара $x_{2}^{*}, x_{2}^{*} p_{N 2}^{-1}$ и $\varkappa_{2}^{*} p_{N 2}^{-1}\left(v_{N 2}^{-1}\right)$, условий (2.4) и формулы ([22, утверждение 29.9]) замены переменных под знаком интеграла Лебега при $N \geqslant 2$ получим:

$$
\begin{aligned}
\int_{G_{2}^{*}} \Delta\left(\gamma_{2}\right) d x_{2}^{*}\left(\gamma_{2}\right) & \geqslant \int_{G_{2}^{*}}\left|\varphi_{N}\left(\gamma_{N 1}, v_{N 2}\left(p_{N 2}\left(\gamma_{2}\right)\right)\right)\right| d x_{2}^{*}\left(\gamma_{2}\right) \\
& =\int_{G_{2}^{*} / L_{N 2}}\left|\varphi_{N}\left(\gamma_{N 1}, v_{N 2}(y)\right)\right| d x_{2} p_{N 2}^{-1}(y) \\
& =\int_{G_{N 2}^{*}}\left|\varphi_{N}\left(\gamma_{N 1}, \gamma_{N 2}\right)\right| d x_{N 2}^{*}\left(\gamma_{N 2}\right) .
\end{aligned}
$$

Тем самым, х.ф. $\varphi_{N}$ абсолютно интегрируема по мере Хаара $x_{N 2}^{*}$, что, в частности, влечет существование (ограниченной) непрерывной версии $d \mathrm{P}_{2} \eta_{N}^{-1} / d \varkappa_{N 2}$ плотности распределения случайной величины $\eta_{N}$ (и, следовательно, открытость в топологии $\mathfrak{U}_{N 2}$ множества $\left.\widetilde{V}_{N}\right)$. Воспользовавшись результатом Зейбелла (формулы (1.15), (1.17)), получим, что 
значение условной х.ф. $\widetilde{\varphi}_{\xi_{N} \mid \eta_{N}}\left(\cdot \mid a_{N}^{0}\right)$, соответствующей непрерывной в смысле слабой сходимости версии $\widehat{\mathbf{P}}_{\xi_{N} \mid \eta_{N}}\left(\cdot \mid v_{N}\right), v_{N} \in \tilde{V}_{N}$, регулярного условного распределения $\xi_{N}$ при условии $\eta_{N}$, определяется при всех $\gamma_{N 1} \in \mathbf{G}_{N 1}^{*}$ формулой:

$$
\begin{aligned}
\tilde{\varphi}_{\xi_{N} \mid \eta_{N}}\left(\gamma_{N 1} \mid a_{N}^{0}\right)= & \int_{G_{N 2}^{*}} \varphi_{N}\left(\gamma_{N 1}, \gamma_{N 2}\right) \gamma_{N 2}\left(-a_{N}^{0}\right) d x_{N 2}^{*}\left(\gamma_{N 2}\right) \\
& \times\left\{\int_{G_{N 2}^{*}} \varphi_{N}\left(e_{N 1}^{*}, \gamma_{N 2}\right) \gamma_{N 2}\left(-a_{N}^{0}\right) d x_{N 2}^{*}\left(\gamma_{N 2}\right)\right\}^{-1},
\end{aligned}
$$

причем знаменатель в (2.9) представляется в виде

$$
\frac{d \mathbf{P}_{2} \eta_{N}^{-1}}{d x_{N 2}}\left(a_{N}^{0}\right)=\int_{G_{N 2}^{*}} \varphi_{N}\left(e_{N 1}^{*}, \gamma_{N 2}\right) \gamma_{N 2}\left(-a_{N}^{0}\right) d x_{N 2}^{*}\left(\gamma_{N 2}\right)
$$

6) Докажем, что, начиная со значения $N \equiv N_{1}$, существует (открытая) окрестность $V_{N}^{\prime}$, содержапцая точку $a_{N}^{0}$ и в каждой точке $v_{N}$ которой совпадают значения $\widehat{\mathbf{P}}_{\xi_{N} \mid \eta_{N}}\left(\cdot \mid v_{N}\right)$ и $\widehat{\mathbf{Q}}_{\xi_{N} \mid \eta_{N}}\left(v_{N} \mid \cdot\right)$ версий регулярного условного распределения случайной величины $\xi_{N}$ при заданной случайной величине $\eta_{N}$. Первая версия определяется согласно (2.9) и негрерывна в точках $v_{N} \in \widetilde{V}_{N}$, а вторая - согласно формуле (1.12) индуцируется версией $\widehat{\mathbf{Q}}_{\theta(N) \mid \eta_{N}}(\cdot \mid \cdot)$ регулярного условного распределения, непрерывной, в смысле слабой сходимости, по первому аргументу в (открытой) окрестности $\widetilde{V}_{N}$. Множество $V_{N}^{\prime}=\widetilde{V}_{N} \cap V_{N}$ является непустым открытым множеством, содержащим точку $a_{N}^{0}$. Пусть при фиксированном значении $N \geqslant N_{1}$ точки $v_{N}, v_{N}(1), \ldots, v_{N}(m), \ldots$ принадлежат $V_{N}^{\prime}$ и $v_{N}(m) \rightarrow v_{N}$ при $m \rightarrow \infty$. Согласно свойству непрерывности рассматриваемых версий при $m \rightarrow \infty$ имеем:

$$
\begin{aligned}
\widehat{\mathbf{P}}_{\xi_{N} \mid \eta_{N}}\left(\cdot \mid v_{N}(m)\right) & \Longrightarrow \widehat{\mathbf{P}}_{\xi_{N} \mid \eta_{N}}\left(\cdot \mid v_{N}\right), \\
\widehat{\mathbf{Q}}_{\xi_{N} \mid \eta_{N}}\left(v_{N}(m), \cdot\right) & \Rightarrow \widehat{\mathbf{Q}}_{\xi_{N} \mid \eta_{N}}\left(v_{N}, \cdot\right) .
\end{aligned}
$$

Отсюда по теореме (см. [24], [43]) о непрерывном соответствии между распределениями и х.ф. для соответствующих версий условных х.ф. при каждом не зависящем от $m$ значении $\gamma_{N 1} \in \mathbf{G}_{N 1}^{*}$ получим соотношения

$$
\begin{array}{ll}
\tilde{\varphi}_{\xi_{N} \mid \eta_{N}}\left(\gamma_{N 1} \mid v_{N}(m)\right) \longrightarrow \tilde{\varphi}_{\xi_{N} \mid \eta_{N}}\left(\gamma_{N 1} \mid v_{N}\right), & m \rightarrow \infty, \\
\widehat{\varphi}_{\xi_{N} \mid \eta_{N}}\left(\gamma_{N 1} \mid v_{N}(m)\right) \longrightarrow \widehat{\varphi}_{\xi_{N} \mid \eta_{N}}\left(\gamma_{N 1} \mid v_{N}\right), & m \rightarrow \infty,
\end{array}
$$

означающие непрерывность в окрестности $V_{N}^{\prime}$ функций $\widetilde{\varphi}_{\xi_{N} \mid \eta_{N}}\left(\gamma_{N 1} \mid \cdot\right)$, $\widehat{\varphi}_{\xi_{N} \mid \eta_{N}}\left(\gamma_{N 1} \mid \cdot\right)$ при каждом фиксированном значении $\gamma_{N 1} \in \mathbf{G}_{N 1}^{*}$. Предположим, что для некоторых значений $\gamma_{N 1}^{0} \in \mathbf{G}_{N 1}^{*}$ и $v_{N}^{0} \in V_{N}^{\prime}$ величина

$$
\varepsilon_{N}\left(\gamma_{N 1}^{0}, v_{N}^{0}\right) \equiv\left|\widetilde{\varphi}_{\xi_{N} \mid \eta_{N}}\left(\gamma_{N 1}^{0} \mid v_{N}^{0}\right)-\widehat{\varphi}_{\xi_{N} \mid \eta_{N}}\left(\gamma_{N 1}^{0} \mid v_{N}^{0}\right)\right|
$$

положительна. Так как $\mathbf{G}_{N 2}$ является локально компактным хаусдорфовым пространством, то (теорема на с. 196 в [7]) семейство замкнутых компактных окрестностей произвольной точки образует - наряду с семейством открытых окрестностей - базу системы всех ее окрестностей.

4 Теорих вероятностей и ее применения, 쇼 1 
В силу непрерывности на открытом множестве $V_{N}^{\prime}$ функций $\varepsilon_{N}\left(\gamma_{N 1}^{0}, \cdot\right)$ и $\left(d \mathbf{P}_{2} \eta_{N}^{-1} / d x_{N 2}\right)(\cdot)$ найдутся замкнутые компактные множества $K_{N}^{\prime}$ и $K_{N}^{\prime \prime}$ соответственно, содержашие точку $v_{N}$, и такие, что значения указанных функций на соответствующем компакте не меньше положительного числа $\delta$. Множество $K=K_{N}^{\prime} \cap K_{N}^{\prime \prime}$ является (замкнутой компактной) окрестностью точки $v_{N}$, так что найдется множество $V_{N}^{0}$ из базы открытых окрестностей этой точки, содержащееся в $K$. Мера Хаара непустого открытого множества положительна ([22, утверждение 54.2 , свойство (ii)]), и, следовательно,

$$
\mathbf{P}_{2} \eta_{N}^{-1}\left(V_{N}^{0}\right)=\int_{V_{N}^{0}} \frac{d \mathbf{P}_{2} \eta_{N}^{-1}}{d \varkappa_{N 2}}\left(v_{N}\right) d \varkappa_{N 2}\left(v_{N}\right) \geqslant \delta \varkappa_{N 2}\left(V_{N}^{0}\right)>0 .
$$

Последнее неравенство противоречит тому, что две версии условной $\mathbf{x} . \boldsymbol{\phi}$. могут отличаться лишь на множестве $\mathbf{P}_{2} \eta_{N}^{-1}$-меры нуль. Тем самым, в окрестности $V_{N}^{0}$ совпадают рассматриваемые версии условной х.ф. (а значит, и соответствующего условного распределения). Следовательно, с учетом (1.13) при $N \geqslant N_{1}$ получаем

$$
\mathbf{P}_{1} \zeta_{N}^{-1}(\cdot)=\widehat{\mathbf{P}}_{\xi_{N} \mid \eta_{N}}\left(\cdot \mid a_{N}^{0}\right),
$$

причем правой части этого равенства соответствует х.ф. (2.9).

7) Для завернения доказательства остается вычислить предельное значение правой части (2.9). Воспользовавшись упомянутыми выше заменами переменных под знаком интеграла Лебега й положив $\gamma_{N 1} \equiv$ $v_{N 1}\left(p_{N 1}\left(\gamma_{1}\right)\right)$ для всех значений $\gamma_{1}$ из смежного класса $v_{N 1}^{-1}\left(\gamma_{N 1}\right)$, получим:

$$
\begin{gathered}
\int_{G_{N 2}^{*}} \varphi_{N}\left(\gamma_{N 1}, \gamma_{N 2}\right) \gamma_{N 2}\left(-a_{N}^{0}\right) d x_{N 2}^{*}\left(\gamma_{N 2}\right) \\
=\int_{G_{2}^{*}} \varphi_{N}\left(v_{N 1}\left(p_{N 1}\left(\gamma_{1}\right)\right), v_{N 2}\left(p_{N 2}\left(\gamma_{2}\right)\right)\right) \\
\quad \times\left(v_{N 2} \odot p_{N 2} \odot \gamma_{2}\right)\left(-a_{N}^{0}\right) d \varkappa_{2}^{*}\left(\gamma_{2}\right) .
\end{gathered}
$$

Согласно (2.8) величина $\left(v_{N 2} \odot p_{N 2} \odot \gamma_{2}\left(-a_{N}^{0}\right)\right.$ равна $\left.\gamma_{2}\right)\left(-a_{N}^{0}\right)$ и постоянна на смежных классах факторгруппы $\mathrm{G}_{2}^{*} / \mathrm{L}_{N 2}$, так что в силу непрерывности $\gamma_{2}$ и условия $(2.5)$ имеем:

$$
\left(v_{N 2} \odot p_{N 2} \odot \gamma_{2}\right)\left(-a_{N}^{0}\right) \longrightarrow \gamma_{2}\left(-a^{0}\right) \quad \text { при } \quad N \rightarrow \infty
$$

для каждого фиксированного $\gamma_{2} \in \mathbf{G}_{2}^{*}$. Учитывая условия д) и е) и применяя теорему Лебега (утверждение 29.12 из [22]) о мажорируемой сходимости, из (2.12) и (2.13) для каждого фиксированного значения $\gamma_{1} \in \mathbf{G}_{1}^{*}$ получим:

$$
\begin{aligned}
& \lim _{N \rightarrow \infty} \int_{G_{N 2}^{*}} \varphi_{N}\left(\gamma_{N 1}, \gamma_{N 2}\right) \gamma_{N 2}\left(-a_{N}^{0}\right) d x_{N 2}^{*}\left(\gamma_{N 2}\right) \\
& =\int_{G_{2}^{*}} \varphi\left(\gamma_{1}, \gamma_{2}\right) \gamma_{2}\left(-a^{0}\right) d x_{2}^{*}\left(\gamma_{2}\right)
\end{aligned}
$$


Полагая в (2.14) $\gamma_{1}=e_{1}^{*}$ и замечая, что $v_{N 1}\left(p_{N 1}\left(e_{1}^{*}\right)\right)=e_{N 1}^{*}$ для всех $N \geqslant 2$, при $N \rightarrow \infty$ выводим:

$$
\begin{gathered}
\int_{G_{N 2}^{*}} \varphi_{N}\left(e_{N 1}^{*}, \gamma_{N 2}\right) \gamma_{N 2}\left(-a_{N}^{0}\right) d x_{N 2}^{*}\left(\gamma_{N 2}\right) \\
\longrightarrow \int_{G_{2}^{*}} \varphi\left(e_{1}^{*}, \gamma_{2}\right) \gamma_{2}\left(-a^{0}\right) d \varkappa_{2}^{*}\left(\gamma_{2}\right)
\end{gathered}
$$

Из условия е) вытекает абсолютная мажорируемость (равномерно по $\left.\gamma_{1} \in \mathbf{G}_{1}^{*}\right)$ по второму аргументу функции $\varphi(\cdot, \cdot)$ функцией $\Delta$. Это, прежде всего, влечет существование (см. формулу (1.15)) ограниченной непрерывной (на множестве (2.24)) версии $d \mathbf{P}_{2} \eta^{-1} / d \varkappa_{2}$ плотности распределения случайной величины $\eta$ по отношению к мере Хаара $\varkappa_{2}$. Поэтому согласно формуле (2.10) и условию (2.6), начиная с некоторого значения $N \equiv N_{2} \geqslant N_{1}$, знаменатель правой части формулы (2.9) отличен от нуля. Учитывая формулы (2.14) и (2.15), из (2.9) для каждого фиксированного значения $\gamma_{1} \in \mathbf{G}_{1}^{*}$ получим:

$$
\begin{aligned}
\lim _{N \rightarrow \infty} \tilde{\varphi}_{\xi_{N} \mid \eta_{N}}\left(\gamma_{1} \mid a_{N}^{0}\right)= & \int_{G_{2}^{*}} \varphi\left(\gamma_{1}, \gamma_{2}\right) \gamma_{2}\left(-a^{0}\right) d x_{2}^{*}\left(\gamma_{2}\right) \\
& \times\left\{\int_{G_{2}^{*}} \varphi\left(e_{1}^{*}, \gamma_{2}\right) \gamma_{2}\left(-a^{0}\right) d x_{2}^{*}\left(\gamma_{2}\right)\right\}^{-1} .
\end{aligned}
$$

Согласно результату Зейбелла (см. пункт 1.4), правая часть формулы (2.16) определяет - с учетом утверждения 1) теоремы - версию $\mathbf{P}_{\xi \mid \eta}$ регулярного условного распределения, непрерывную (в смысле слабой сходимости) при всех $a^{0} \in V$. Утверждение 2) теоремы следует из формул (2.11) и (2.16).

З а м е ч а н и е. В работах [17], [18] автора намечены доказательства утверждений теоремы для частного случая:

$$
G_{N j} \leqslant G_{N+1, j} ; \quad N=2,3 \ldots \quad(j=1,2) .
$$

\section{§3. Некоторые применения}

3.1. Сходимость условных распределений. По существу, доказательство теоремы не использует представление случайных величин $\xi_{N}$ и $\eta_{N}$ в форме сумм. Вариант (с ограничениями (2.17)) следующего утверждения приведен в работах [16], [17].

Следствие 1. Предположим, что случайные величины $\xi_{N}$ и $\eta_{N}$ не обнзательно имеют аддитивную форму (2.3), но выполняются условия в)-е) теоремы §2. Тогда имеет место утверждение 1) этой теоремы и в асимптотике (2.5) при справедливости условия (2.6) последовательность $\widehat{\mathbf{P}}_{\xi_{N} \mid \eta_{N}}\left(\cdot \mid a_{N}^{0}\right), N \geqslant 2$, определяемая правой частью (2.9), слабо сходится $\boldsymbol{x}$ значению $\widehat{\mathbf{P}}_{\xi \mid \eta}\left(\cdot \mid a^{0}\right)$ версии $\widehat{\mathbf{P}}_{\xi \mid \eta}(\cdot \mid \cdot)$ регулярного условного распределения, непрерывной (ө смысле слабой сходимости) 
по второму аргументу на множестве (2.7) и определяемой правой частью формулы (2.16).

Доказательство следует из доказательства теоремы.

Применительно к действительнозначным (или векторным с действительными компонентами) случайным суммам $\xi_{N}$ и $\eta_{N}$ c $l=0$, в наших обозначениях, задача сходимости условных распределений рассматривалась Парзеном [44], Стэком [45], Д. М. Чибисовым [33], Михелем [40], Хольстом [37] и другими авторами; при этом всегда, исключая работы $[13],[15],[17]$ автора, отдельно изучались случаи абсолютно непрерывных (по мере Лебега) и решетчатых распределений, и, как правило, в качестве предельного распределения рассматривалось нормальное. Полученный Стэком [45, теорема 2.1] результат о предельном законе общего вида носит «дескриптивный» характер и не дает явной формулы для предельного распределения.

Представление случайных величин $\xi_{N}$ и $\eta_{N}$ в виде сумм позволяет воспользоваться результатами о предельных законах: а) для $m$-зависимых случайных величин - при $l>0$ (см. $[5$, с. 469], и $[1, \S 20])$ и б) для независимых случайных величин - при $l=0$ (см. [2], [30], [46] - в случае действительнозначных $\xi_{N}, \eta_{N}$, и [42], [43] - в случае LCA-значных $\left.\xi_{N}, \eta_{N}\right)$. Важным классом предельных распределений сумм независимых случайных величин являются безгранично делимые распределения.

3.2. Безгранично делимые распределения на LCA-группах и предельные распределения разделимых статистик. Пусть $\mathbf{G}=$ $(G,+, \mathfrak{U})$ - сепарабельная метрическая абелева группа, $\mathfrak{B}$ - наименьшая $\sigma$-алгебра, содержашая топологию $\mathfrak{U}$, и $M(\mathbf{G})-$ множество всех вероятностных мер, определенных на пространстве $(G, \mathfrak{B})$. Сверткой двух мер $\mu, \nu \in M(\mathbf{G})$ называется функция $\mu * \nu$ (или $\nu * \mu$ ), определяемая для всех $B \in \mathfrak{B}$ формулой:

$$
(\mu * \nu)(B)=\int_{G} \mu(B-x) d \nu(x)=\int_{G} \nu(B-x) d \mu(x)=(\nu * \mu)(B) .
$$

Коррегтность определения свертки доказывается стандартным образом (см., например, $[22$, формулу (36.1)], и $[24$, с. 8]) с использованием указанного выше наличия второй аксиомы счетности для $\mathbf{G}$. Если $\delta_{g}-$ мера, сосредоточенная в точке $g \in G$, то очевидно, что $\left(\mu * \delta_{g}\right)(B)=$ $\left(\delta_{g} * \mu\right)(B)=\mu(B-g)$ для всех $B \in \mathfrak{B}$. Мера $\mu \in M(\mathbf{G})$ называется идемпотентной, если $\mu * \mu=\mu$. Пусть $X$ и $Y-$ независимые случайные величины, принимағоие значения в $\mathbf{G}$ и имеющие распределения $\mu_{X}$ и $\mu_{Y}$ соответственно. Тогда (см. [24, с. 8-10]) сумма $Z=X+Y$ является случайной величиной и $\mu_{Z}=\mu_{X} * \mu_{Y}$. Везде далее $\mathbf{G}-$ LCA-группа. Распределение $\mu \in M(\mathbf{G})$ называется [42, с. 77, определение 4.1] безгранично делимьм, если для каждого натурального $n$ сушествуют распределение $\mu_{n} \in M(\mathbf{G})$ и элемент $g_{n} \in \mathbf{G}$, такие, что $\mu$ является сверткой $n$ распределений $\mu_{n}$ и распределения, сосредоточенного в $g_{n}$. Наличие в 
этом определении (в отличие от классического, когда $\mathbf{G}=\mathbf{R}^{a} \times \mathbf{Z}^{b}$ с целыми неотрицательными степенями $a$ и $b$, сумма которых не меныше 1) элемента $g_{n}$ связано с отсутствием, вообще говоря, «делимости» элементов группы G. Характеристическая функция $\widehat{\mu}$ безгранично делимого распределения $\boldsymbol{\mu}$ равна 0 тогда и только тогда (см. $[42$, с. 78 , теорема $4.2]$ ), когда $\mu$ представляется в виде свертки какого-либо распределения с невырожденным идемпотентным распределением (в таких случаях говорят, что $\mu$ имеет идемпотентный делитель). Для компактной подгруппы, $\mathbf{H} \subseteq \mathbf{G}$ мера Хаара $\boldsymbol{x}$ является конечной (так что ее можно нормализовать) и безгранично делимой; кроме того, $\widehat{x}(\gamma)=1$, если $\gamma$ принадлежит аннулятору $\mathbf{H}$, и $\widehat{\boldsymbol{\kappa}}(\gamma)=0$ - в противном случае. Следовательно, если распределение $\mu \in M(\mathbf{G})$ - безгранично делимо, то $\mu=\lambda * \nu$, где $\lambda$ нормализованная мера Хаара некоторой компактной подгруппы в $\mathbf{G}$ и $\nu$ - безгранично делимое распределение без идемпотентных делителей.

Рассмотрим примеры безгранично делимых распределений. Пусть F - произвольная вполне конечная мера на G. Paспределение е(F), ассоииированное $c$ F, определяется (см. [43, определение 4.2]) формулой:

$$
e(\mathbf{F})=\exp \{-\mathbf{F}(\mathbf{G})\}\left[1+\mathbf{F}+\frac{\mathbf{F}^{2}}{2 !}+\cdots+\frac{\mathbf{F}^{n}}{n !}+\cdots\right],
$$

где 1 - мера единичной массы, сосредоточенная в нейтральном элементе е группы $\mathbf{G}$, и $\mathbf{F}^{n}-n$-кратная свертка меры $\mathbf{F}$. Характеристическая функция меры (3.1) для всех $\gamma \in \mathbf{G}^{*}$ определяется формулой:

$$
\widehat{\boldsymbol{e}}(\mathbf{F})(\gamma)=\exp \left\{\int_{G}[\gamma(g)-1] d \mathbf{F}(g)\right\} .
$$

Отсюда вытекает, что для любых двух вполне конечных мер $\mathbf{F}_{1}$ и $\mathbf{F}_{2}$ $e\left(\mathbf{F}_{1}\right) * e\left(\mathbf{F}_{2}\right)=e\left(\mathbf{F}_{1}+\mathbf{F}_{2}\right)$, что, в частности, для каждого натурального $n$ влечет равенство: $e(\mathbf{F})=[e(\mathbf{F} / n)]^{n}$. Следовательно, $e(\mathbf{F})$ является безгранично делимым распределением; распределения типа (3.2) иногда называются обобщенными распределениями Пуассона.

Распределение $\boldsymbol{\mu}$ на LCA-группе G называется (см. [43, определение 6.1]) аауссовским в смысле Партасарати или Р-гауссовским (или просто гауссовским), если $\mu$ - безгранично делимо и при любой факторизации: $\mu=e(\mathbf{F}) * \alpha$, распределение $\alpha$ безгранично делимо и мера $\mathbf{F}$ cocpeдоточена в нейтральном элементе е группы G. Это определение совпадает с классическим определением нормального закона, когда $\mathbf{G}=\mathbf{R}^{n} \mathbf{c}$ любым натуральным $n$. Характеристическая функция $\widehat{\mu}$ P-гауссовского закона на LCA-групле $\mathbf{G}$ для каждого $\gamma \in \mathbf{G}^{*}$ определяется формулой:

$$
\widehat{\mu}(\gamma)=\gamma\left(g_{0}\right) \exp \{-\varphi(\gamma)\}
$$

где $g_{0}$ - некоторый фиксированный элемент $G$ и $\varphi-$ неотрицательная непрерывная функция, удовлетворяющая для всех $\gamma_{1}, \gamma_{2} \in \mathbf{G}^{*}$ равенству

$$
\varphi\left(\gamma_{1}+\gamma_{2}\right)+\varphi\left(\gamma_{1}-\gamma_{2}\right)=2\left[\varphi\left(\gamma_{1}\right)+\varphi\left(\gamma_{2}\right)\right]
$$


(если $\gamma\left(g_{0}\right)=1$, то соответствующее гауссовское распределение называется симметричным). Среди других обобщений нормального распределения на LCA-группы наиболее известно (см., например, $[29,5.3]$ ) гауссовское распределение в смысле Бернитейна (для краткости, Вгауссовское распределение); в отличие от В-гауссовского, Р-гауссовское распределение не имеет идемпотентных делителей (соотношения между Р- и В-гауссовскими мерами приводятся в $[29,5.3]$, и в [27]).

Распределение $\mu \in M(\mathbf{G})$ называется [28] распределением Коши, если х.ф. $\widehat{\mu}$ для каждого $\gamma \in \mathbf{G}^{*}$ определяется формулой:

$$
\widehat{\mu}(\gamma)=\gamma\left(g_{0}\right) \exp \left\{-(\varphi(\gamma))^{1 / 2}\right\},
$$

где $g_{0}$ - некоторый элемент из $G$ и функция $\varphi$ такого же вида, как в (3.3). Для каждого натурального $n$ х.ф. (3.4) удовлетворяет соотношению: $\widehat{\mu}(n \gamma)=(\widehat{\mu}(\gamma))^{n}$, так что распределение Коши является безгранично делимым.

На декартовом произведении пространств $\mathbf{G} \times \mathbf{G}^{*}$ сушествует (см. [43, с. 119-120]) действительная функция $g$, обладағшая свойствами:

1) $g$ - непрерывная функция;

2) $\sup _{g \in G} \sup _{\gamma \in K}|g(g, \gamma)|<+\infty$ для каждого компактного множества $K \subset \mathbf{G}^{*}$;

3) $g\left(g, \gamma_{1}+\gamma_{2}\right)=g\left(g, \gamma_{1}\right)+g\left(g, \gamma_{2}\right)$ для каждого $g \in G$ и всех $\gamma_{1}, \gamma_{2} \in$ $\mathbf{G}^{*}$ и $g(-g, \gamma)=-g(g, \gamma)$ для всех $g \in G$ и всех $\gamma \in \mathbf{G}^{*}$;

4) для любого компактного множества $K \subset \mathbf{G}^{*}$ существует окрестность $V_{K}$ нейтрального элемента е группы $\mathbf{G}$, такая, что $\gamma(g)=$ $\exp \{i g(g, \gamma)\}$ для всех $g \in G$ и всех $\gamma \in K$;

5) если $g$ стремится к е, то $g$ стремится к нулю равномерно по всем $\gamma \in K$ для любого компактного множества $K \subset \mathbf{G}^{*}$. Функция $g$ называется (см. [29, определение 5.1.7]) покальным скалярным произведением или чентрирующей функиией. Примеры функции $g$ приведены в [42, c. $87-88]$, и в $[24$, с. 26$]$.

Для х.ф. $\widehat{\mu}$ безгранично делимого распределения $\mu$ имеет место (см. [42, теорема 7.1, и следствие 7.1]) аналог канонического представления Леви-Хинчина [30], [2]:

$$
\widehat{\mu}(\gamma)=\gamma\left(g_{0}\right) \widehat{\lambda}(\gamma) \exp \left\{\int_{G}[\gamma(g)-1-i g(g, \gamma)] d F(g)-\varphi(g)\right\}
$$

для всех $\gamma \in \mathbf{G}^{*}$. В формуле (3.5) $g_{0}$ - некоторый фиксированный элемент группы $\mathbf{G}, \varphi$ и $g-$ упомянутые выше функции; $\lambda$ - нормализованная мера Хаара компактной подгруппы (возможно тривиальной) группы $\mathrm{G} ; \boldsymbol{F}-\sigma$-конечная мера на $\mathfrak{B}$ с конечной «массой» вне любой окрестности нейтрального элемента е группы $\mathbf{G}$, удовлетворяющая для всех $\gamma \in \mathbf{G}^{*}$ соотношению $\int_{G}[1-\operatorname{Re} \gamma(g)] d F(g)<+\infty$.

При справедливости (3.5) говорят (см. [42, с. 109]), что мера $\mu$ имеет представление $(g, F, \varphi)$. Это представление не является единственным, если мера $\mu$ имеет нетривиальный идемпотентный делитель (так как для 
любой меры $\nu$, сосредоточенной на носителе $\lambda$, имеем: $\lambda * \nu=\lambda$ ). Пусть $\left(g^{\prime}, F^{\prime}, \varphi^{\prime}\right)$ и $\left(g^{\prime \prime}, F^{\prime \prime}, \varphi^{\prime \prime}\right)$ - два представления одного и того же безгранично делимого распределения $\mu$ без идемпотентных делителей. Тогда (см. [42, теорема 8.1]) $\varphi^{\prime}=\varphi^{\prime \prime}$ и знаковая мера $F^{\prime}-F^{\prime \prime}$ тождественно равна нулю на дополнении аннулятора компоненты нейтрального элемента $\mathrm{e}^{*}$ группы $\mathbf{G}^{*}$. Этот аннулятор является (см. $[42$, с. 111 , замечание 1]) наименьшей замкнутой подгруппой, содержащей все компактные подгруппы группы G, или, что эквивалентно, он является группой всех компактных элементов в $\mathbf{G}^{*}$. Указанное обстоятельство отражает роль компактных подгрупп группы $\mathbf{G}$ (или, что эквивалентно, роль несвязности двойственной групाы $\mathbf{G}^{*}$ ) в неединственности представления (2.10). Представление (2.10) безгранично делимого распределения без идемпотентных делителей является (см. [42, с. 112]) единственным тогда и только тогда, когда каждый элемент любой компактной подгруппы группы $\mathbf{G}$ представляет собой элемент порядка 2.

Предположим, что $\mathbf{G}$ - вполне несвязная группа и безгранично делимое распределение $\mu \in M(\mathbf{G})$. Для такой группы функция $g$ тождественно равна нулю и, кроме того (см. [42, с. 109, замечание 1]), функция $\varphi$ также тождественно равна нулю; в этом случае вместо (3.5) для всех $\gamma \in \mathbf{G}^{*}$ имеем:

$$
\widehat{\mu}(\gamma)=\gamma\left(g_{0}\right) \hat{\lambda}(\gamma) \exp \left\{\int_{G}[\gamma(g)-1] d F(g)\right\} .
$$

Представление (3.6) справедливо, в частности, и для конечной группы $\mathbf{G}$ - для нее топология $\mathfrak{U}$ дискретна.

Пусть теперь $\mathbf{G}=\mathbf{R}^{n}$. Тогда х.ф. $\hat{\mu}$ безгранично делимого распределения $\boldsymbol{\mu}$ допускает (см., например, [46]) представление:

$$
\begin{aligned}
\widehat{\mu}(\mathrm{t})=\exp \left\{i \mathrm{a}^{\prime} \mathrm{t}-\right. & \frac{1}{2} \mathrm{t}^{\prime} \Sigma \mathrm{t} \\
& \left.+\int_{\mathbf{R}^{n}} \exp \left[i \mathrm{t}^{\prime} \mathbf{x}-1-\frac{i \mathrm{t}^{\prime} \mathbf{x}}{1+\mathbf{x}^{\prime} \mathbf{x}}\right] d F(\mathbf{x})\right\}
\end{aligned}
$$

где $\mathbf{t}, \mathbf{a}, \mathbf{x} \in \mathbf{R}^{n}$ - вектор-столбцы, $\Sigma$ - неотрицательная матрица $n$-го порядка и $F-\sigma$-конечная мера на $\mathfrak{B}_{\mathbf{R}^{n}}$ такая, что при $|\mathbf{x}|=\left(\mathbf{x}^{\prime} \mathbf{x}\right)^{1 / 2}$

$$
\int_{|\mathbf{x}|<1}|\mathbf{x}|^{2} d F(\mathbf{x})<+\infty, \quad \int_{|\mathbf{x}| \geqslant 1} d F(\mathbf{x})<+\infty .
$$

Отметим, что представление (3.7) для каждого безгранично делимого распределения является единственным (в силу отсутствия на $\mathbf{R}^{n}$ нетривиальных компактных подгрупा).

Пусть $\left\{\mu_{n 1}, \ldots, \mu_{n k_{n}}\right\}, n>1,-$ последовательность серий независимых в каждой серии распределений $\mu_{n k} \in M(\mathbf{G})$ и пусть

$$
k_{n} \rightarrow \infty \quad \text { при } n \rightarrow \infty \text {. }
$$

Предположим, что для этой последовательности имеет место условие 
равномерной асимптотической малости (см. [42, определение 5.1]):

$$
\lim _{n \rightarrow \infty} \sup _{1 \leqslant k \leqslant k_{n}} \mu_{n k}\left(U^{c}\right)=0
$$

где $U^{c}$ - дополнение произвольной не зависяшей от $n$ окрестности $U$ нейтрального элемента е $\in \mathbf{G}$. Эквивалентное определение в терминах Х.Ф. $\widehat{\mu}_{n k}$, соответствующей мере $\mu_{n k}$, имеет вид (см. [42, с. 82]):

$$
\lim _{n \rightarrow \infty} \sup _{1 \leqslant k \leqslant k_{n}} \sup _{\gamma \in K}\left|\widehat{\mu}_{n k}(\gamma)-1\right|=0
$$

для каждого компактного множества $K \subseteq \mathbf{G}^{*}$. Справедливо (см. [42, теорема 5.1]) следующее обобщение результата А. Я. Хинчина [30], [2]: если выполняются условия (3.8), (3.9) и

$$
\mu_{n} \equiv \mu_{n 1} * \cdots * \mu_{n k_{n}} \equiv \prod_{k=1}^{k_{n}} \mu_{n k} \Rightarrow \mu
$$

то $\mu$ - безгранично делимое распределение; обратно, если в (3.11) $\mu$ безгранично делимое распределение, то существует [24, с. 27] входящая в (3.11) последовательность серий $\left\{\mu_{n k}\right\}$, удовлетворяющая условию равномерной асимптотической малости.

Имеет место (см. [42, следствие 9.2] и [24, теорема 4.11]) следующее обобщение центральной предельной теоремы. Пусть выполняются условия (3.8), (3.9) и (3.11), причем распределение $\mu$ не имеет идемпотентных делителей. Распределение $\mu$ является Р-гауссовским тогда и только тогда, когда $\lim _{n \rightarrow \infty} \sum_{k=1}^{k_{n}} \mu_{n k}\left(U^{c}\right)=0$ для любой окрестности $U$ элемента е $\in \mathbf{G}$.

Пусть $\mathbf{G}$ - компактная сепарабельная метрическая (возможно, некоммутативная) группа. Согласно Б. М. Клоссу [8] (см. также с. 1920 в [24]) группа $\mathbf{G}$ обладает свойством (K), если, какова бы ни была последовательность распределений $\left\{\mu_{n} \in M(\mathbf{G})\right\}$, существует такая последовательность элементов $\left\{g_{n} \in \mathbf{G}\right\}$, что последовательность $\left\{\mu_{1} * \cdots *\right.$ $\left.\mu_{n} * \delta_{g_{n}}\right\}$ имеет слабый предел при $n \rightarrow \infty$. Справедлив принадлежаший Б. М. Клоссу (см. также теорему 3.4 из [24]) обиий приниип сходимости: любая компактная группа, удовлетворяющая первой аксиоме счетности, обладает свойством (K); если LCA-группа обладает свойством $(\mathrm{K})$, то она компактна.

3 а м е ч а и е. Чтобы выполнялось предположение д) теоремы, мы должны, вообще говоря, от случайных величин $\xi_{N}$ и $\eta_{N}$ перейти к случайным величинам $\xi_{N}^{0}=v_{N}^{(1)}\left(\xi_{N}\right), \eta_{N}^{0}=v_{N}^{(2)}\left(\eta_{N}\right) ; N \geqslant 2$, где $v_{N}^{(j)}: \mathbf{G}_{j} \rightarrow \mathbf{G}_{j}(j=1,2)$ - некоторые топологические автоморфизмы; при этом условие: $\eta_{N}=a_{N}$ меняется на условие: $\eta_{N}^{0}=a_{N}^{0}$. В случае: $\mathbf{G}_{j}=\mathbf{R}^{b_{j}} \times \mathbf{Z}^{c_{j}} ; b_{j}+c_{j} \geqslant 1(j=1,2)$, преобразования $v_{N}^{(j)} ; j=1,2$, представляют собой определенные (см., например, работы [2], [5], [30], [46]) нормировки, с помощью которых распределения случайных величин $\xi_{N}$ и $\eta_{N}$ концентрируются (с ростом $N$ ) около начала координат. Если же 
$\mathbf{G}_{\boldsymbol{j}}, \boldsymbol{j}=1,2$, - компактные LCA-группы, то, согласно предыдущему абзацу, любую «нарастающую» последовательность сумм независимых случайных величин со значениями в таких группах можно «сдвинуть» с помошью соответствующей последовательности $\left\{g_{N}^{(j)} \in \mathbf{G}_{j}, N \geqslant 2\right\}$ неслучайных элементов, в результате чего преобразованная последовательность будет иметь предельное распределение (при этом $a_{N}^{0}=a_{N}-g_{N}^{(2)}$, $N \geqslant 2$ ). Если, в частности, суммируются одинаково распределенные случайные величины, то предельное распределение суммы является мерой Хаара некоторой подгруппы, определяемой по носителю распределения слагаемых. Например, если $S$ - группа врашений окружности ( $S$ топологически изоморфна $T$ ), то распределение суммы случайных величин слабо сходится к равномерному распределению на окружности, за исключением того случая, когда значениями слагаемых могут быть, с вероятностью 1, только вершины вписанного в указанную окружность многоугольника - это результат ПІ. Леви (см. ссылку [10] к библиографии работы [24]). Случай «элементарных» LCA-групп (см. II. 1.1): $\mathbf{G}_{j}=\mathbf{R}^{b_{j}} \times \mathbf{Z}^{c_{j}} \times \mathbf{T}^{d_{j}} \times \mathbf{F}_{j} ; j=1,2$, является комбинацией рассмотренных выше случаев (отметим, что, согласно теореме А. Н. Тихонова (см. [7, с. 192]), произведение компактных групп остается компактной - в топологии произведения - группой).

В ситуации же общих LCA-групп важную роль играет «сведение» к суммированию «равномерно асимптотически малых» случайных величин (см. формулы (3.9) и (3.10)).

Следствие 2. Предположим, что: а) в формуле (2.1) $l=0$ при всех $N \geqslant 2 ;$ б) выполиялотся условия теоремы; в) имеет место условие (3.10), в котором $n=k_{n}=N, \gamma=\left(\gamma_{1}, \gamma_{2}\right), K$ - компактное множество из $\mathbf{G}_{1}^{*} \times \mathbf{G}_{2}^{*}$, величина $\widehat{\mu}_{n k}(\gamma)$ заменяется на $\varphi_{N}\left(v_{N 1}\left(p_{N 1}\left(\gamma_{1}\right)\right)\right.$, $\left.v_{N 2}\left(p_{N 2}\left(\gamma_{2}\right)\right)\right)$. Тогда справедливы утверждения теоремы с предельной характеристической функчией $\varphi$, определяемой формулой вида (3.5) с заменами $\mathbf{G}$ на $\mathbf{G}_{1} \times \mathbf{G}_{2} u \mathbf{G}^{*} \mu a \mathbf{G}_{1}^{*} \times \mathbf{G}_{2}^{*}$.

Д о к а з те л ь с в о. Согласно предположению д), утверждению 1) теоремы и теореме о непрерывном взаимно однозначном соответствии между распределениями и х.ф., совместное распределение случайных величин $\xi_{N}$ и $\eta_{N}$ слабо сходится к распределению с х.ф. $\varphi$. В силу теоремы 5.2 из [43] (см. также теорему 4.4 из [24] и теорему 5.2 из [42]) условие в) гарантирует безграничную делимость х.ф. $\varphi$, что в свою очередь, влечет (см. $[43, \S \S 7,8]$ ) для $\varphi$ представление вида $(3.5)$ с указанными выше замечаниями.

\section{СПИСОК ЛИТЕРАТУРЫ}

1. Биллинасли П. Сходимость вероятностных мер. М.: Наука, 1977, 352 с.

2. Гкеденко $Б$. В., Колмогоров $A$. $H$. Предельные распределения для сумм незави- 
симых случайных величин. М.: Гостехиздат, 1949, 264 с.

3. Гнеденко Б. В., Кудлаев Э. М. О случайных величинах, обусловленных суммами независимых случайных величин. - Вестинх МГУ, сер. матем. и мех., 1995, № 1 , c. 23-31.

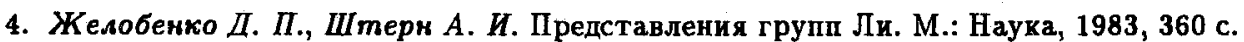

5. Нбрагимов И. А., Лияяик Ю. В. Независимые и стационарно связанные случайные величины. М.: Наука, 1965, 524 с.

6. Иөанов В. А., Иөченко Г. И., Медведев Ю. И. Дискретные задачи теории вероятностей. - Итоги науки и техники, сер. Теория вероятн. Матем. статист. Теорет. киберн., 1984, т. 23, с. 3-60.

7. Келяи Дљс. Общая топология. М.: Наука, 1981, 383 с.

8. Клосс $Б$. $M$. Предельные распределения на бикомпактных абелевых группах. Теория вероятн. и ее примен., 1961, т. VI, в. 3, с. 392-421.

9. Колчия $B$. $Ф$. Один класс предельных теорем для условных распределений. Литов. матем. сб., 1968, т. 8, № 1, с. 53-63.

10. Колчия $B$. Ф. Случайные отображения. М.: Наука, 1984, 206 с.

11. Колчия B. Ф., Севастьянов Б. А., Чистяков В. П. Случайные размещения. М.: Наука, 1976, 223 c.

12. Кудлаев Э. $M$. Асимптотическая нормальность статистик некоторых классов критериев согласия и однородности. - Докл. АН СССР, 1978, т. 240, № 3, с. 534537.

13. Кудлаев Э. М. О предельных условных распределениях сумм случайных величин. - Теория вероятн. и ее примен., 1984, т. XXIX, № 4, с. 743-752.

14. Кудлаев Э. М. Предельные теоремы для одного класса разделимых статистик. Теория вероятн. и ее примен., 1985 т. XXX, № 1, с. 170-174.

15. Кудлаеө Э. М. Об условиях слабой сходимости распределений разделимых статистик. - Матем. заметки, 1986, т. 40, № 6, с. 762-769.

16. Кудлаев Э. М. О сходимости условных распределений случайных элементов со значениями в локально компактных абелевых группах. - В сб.: Вероятностные задачи дискретной математики. М.: Изд-во Московского ин-та электронного машиностр., 1987, с. 73-80.

17. Кудлаев Э. М. Разделимые статистики. - Итоги науки и техники, сер. Теория вероятн. Матем. статист. Теорет. киберн., 1988, т. 27, с. 97-149.

18. Кудлаев Э. М. О суммировании некоторых зависимых случайных величин со значениями в локально компактных абелевых группах. - Изв. вузов, сер. матем., 1989 , № 2, с. $41-46$.

19. Кудлаев Э. М. Классификация разделимых статистик. - В сб.: Вероятностные процессы и их приложения. М.: Изд-во Московского ин-та электронного машиностр., 1989, с. 101-114.

20. Кудлаев Э. М. О слабой сходимости распределений разделимых статистик. Тезисы докл. Второй Всероссийской школы-коллоквиума по стохастическим методам (Йошкар-Ола, 18-23 декабря 1995 г.). М.: ТВП, 1995, с. 83-84.

21. Моррис $C$. Двойстненность Понтрягина и строение локально компактных абелевых групп. М.: Мир, 1980, 103 с.

22. Партасарати $K$. Введение в теорию вероятностей и теорию меры. М.: Мир, 1983.

23. Поктрягия $Л$. С. Непрерывные группы. М.: Наука, 1984, 520 с.

24. Сазоков $B$. В., Тутубалия $B$. $Н$. Распределения вероятностей на топологических rруппах. - Теория вероятн. и ее примен., 1966, т. XI, в. 1, с. 3-56.

25. Севастьяков $B$. A. Сходимость к гауссовскому и пуассоновскому процессам распределения числа «пустых ящиков» в классической задаче о дробинках. - Теория вероятн. и ее примен., 1967, т. XII, в. 1, с. 144-154.

26. Севастьянов Б. А. Предельный закон Пуассона в схеме сумм зависимых величин. - Теория вероятн. и ее примен., 1972, т. XVII, в. 4, с. 733-738. 
27. Фельдман Г. М. Гауссовские распределения в смысле Бернштейна на групдах. Теория вероятн. и ее примен., 1986, т. XXXI, в. 1, с. 47-58.

28. Фельдман $Г$. М. Распределение Коши на абелевых группах и его харахтеризация. Теория вероятн. и ее примен., 1991, т. 36, в. 4, с. 711-743.

29. Хейер $X$. Верохтностные меры на локально компактных группах. М.: Мир, 1981, $701 \mathrm{c}$.

30. Хиниия А. Я. Предельные законы для сумм независнмых случайных величин. М.-Л.: ОНТИ, 1938, 116 c.

31. Barbour A. D., Holst L. Some applications of the Stein-Chen method for proving Poisson convergence. - Adv. Appl. Probab., 1989 v. 21, № 1, p. 74-91.

32. Bartlett $M$. $S$. The characteristic function of a conditional statistic. - J. London Math. Soc., 1938, v. 13, p. 62-67.

33. Chibisov $D . M$. On the normal approximation for certain class of statistics. - In: Proc. of the Sixth Berkeley Symposium on Mathematical Statistics and Probability Theory, v. 1, Los Angeles: Univ. of California Press, 1972, p. 153-174.

34. Darling $D$. $A$. On a class problems related to the random division of an interval. Ann. Math. Statist., 1953, v. 24, № 2, p. 239-253.

35. Hall $P$. Limit theorems for sums of general functions of spacings. - Math. Proc. Cambridge Philos. Soc., 1984, v. 96, p. 517-532.

36. Holst $L$. Two conditional limit theorems with applications. - Ann. Statist., 1979, v. 7, № 3, p. 551-557.

37. Holst $L$. Some conditional limit theorems in exponential families. - Ann. Probab., 1981 , v. 9, № 5, p. 818-830.

38. Kudlaev E. M. On an existence theorem for decomposable statistics. - Beportностные методы дискретной математики. Moscow/Utrecht: TBI/VSP, 1994, p. 271286.

39. Le Cam $L$. Une théorème sur la division d'une intervalle par des points pres au hasard. - Publ. Inst. Statist. Univ. Paris, 1958, v. 7, № 3-4, p. 7-16.

40. Michel $R$. Asymptotic expansions for conditional distributions. - J. Multivariate Anal., 1979, v. 9, № 3, p. 393-400.

41. Morris $C$. Central limit theorems for multinomial sums. - Ann. Statist., 1975, v. 3, № 1 , p. 165-188.

42. Parthasarathy K. R. Probability Measures on Metric Spaces. New York: Academic Press, 1967.

43. Parthasarathy K. R., Ranga Rao $R$., Varadhan $S$. $R$. S. Probability distributions on locally compact abelian groups. - Illinois J. Math., 1963, v. 7, № 2, p. 337-369.

44. Parzen $E$. On uniform convergence of families of sequences of random variables. Univ. Calif. Publ. Statist., 1954, v. 2, p. 23-54.

45. Steck G. P. Limit theorems for conditional distributions. - Univ. Calif. Publ. Statist., 1957 , v. 2, № 12, p. 237-284.

46. Takano $K$. Central convergence criterion in the multidimensional case. - Ann. Inst. Statist. Math., 1956, v. 7, № 2, p. 95-102.

47. Wicksell $S$. $P$. On correlation functions of type III. - Biometrika, 1933, v. 25, № 1 , p. 121-133.

48. Yeh J. Inversion of conditional expectations. - Pacific J. Math., 1974, v. 52, № 2, p. 631-640.

49. Zabell $S$. Continuous versions of regular conditional distributions. - Ann. Probab., 1979 , v. 7 , № 1, p. 159-165. 